\title{
Frauenfördernde Massnahmen in den Sahelländern
}

Frauenförderung bei HEKS

Marie-Thérèse Roggo

\section{CpenEdition}

\section{Journals}

Electronic version

URL: http://journals.openedition.org/sjep/1093

DOI: 10.4000/sjep.1093

ISSN: 1663-9677

Publisher

Institut de hautes études internationales et du développement

\section{Printed version}

Date of publication: 1 décembre 1989

Number of pages: 183-192

ISSN: 1660-5926

\section{Electronic reference}

Marie-Thérèse Roggo, «Frauenfördernde Massnahmen in den Sahelländern », Schweizerisches Jahrbuch für Entwicklungspolitik [Online], 8 | 1989, Online erschienen am: 13 März 2013, abgerufen am 08 September 2020. URL : http://journals.openedition.org/sjep/1093 ; DOI : https://doi.org/10.4000/ sjep.1093 


\title{
Frauenförderung bei HEKS
}

\section{Frauenfördernde Massnahmen in den Sahelländern}

\author{
Marie-Thérèse Roggo
}

Bekanntlich gehören die Sahelstaaten (1) zu den ärmsten Ländern der Welt. Wiederholte Dürrekatastrophen hatten in den letzten fünfzehn Jahren verheerende ökologische, wirtschaftliche und soziale Auswirkungen für die Menschen der Sahelzone gebracht. Das Voranschreiten der Verwüstung als Folge der Zerstörung des ökologischen Gleichgewichts (Abholzung, Überweidung, Ausdehnung des Weidelands auf Kosten des Ackerlands, Auslassen der Brache, etc.), eine ungerechte interne Verteilung der Ressourcen und eine auf den Export konzentrierte Landwirtschaftspolitik machen es den Kleinbäuerinnen und -bauern, welche $80 \%$ der Bevölkerung ausmachen, sehr schwer, ihre Lebensgrundlagen zu verbessern und eine eigenständige Entwicklung zu bestimmen.

In der komplexen und problematischen Situation der Sahelländer sind die Frauen am meisten betroffen, weil sich die negativen Konsequenzen der Politik, der Wirtschaft und der sozio-kulturellen Strukturen direkt auf die Frauen auswirken. Die schwere Wirtschaftskrise Afrikas trifft die Frauen ganz besonders. Obwohl die Verschuldung der Sahelländer in absoluten Zahlen relativ klein ist, wirkt sich diese dramatisch auf die Bevölkerung aus, weil die Erwirtschaftung von Devisen schwierig ist. Es mutet sonderbar an, dass in Ländern, in denen die Selbstversorgung noch längst nicht erreicht ist, die Bauern und Bäuerinnen für den Export produzieren müssen und auf den besten Böden Monokulturen wie z.B. Baumwolle und Erdnüsse angepflanzt werden. Als Hauptproduzentinnen in der Landwirtschaft und als Verantwortliche für die Ernährung der Familie sind die Frauen von dieser Landwirtschaftspolitik unmittelbar betroffen. Mit der Ein- 
gliederung in die Marktwirtschaft und der Modernisierung in Richtung extensiver Landwirtschaft verloren die Frauen zunehmend ihren Einfluss im traditionellen Arbeits- und Einkommensbereich, im Selbstversorgungsanbau, im Handwerk und im Kleinhandel. Die markt- und wachstumsorientierte Landwirtschaft bedeutet für die ohnehin schon voll ausgelasteten Frauen eine zusätzliche Arbeitsbelastung.

In direktem Zusammenhang mit der Schuldenkrise, der Rückzahlung von Exportkrediten und den Strukturanpassungsprogrammen des IWF und der Weltbank, steht auch die Basisgesundheit der Bevölkerung. Einer der traditionellen Hauptinhalte in den Gesundheitsprogrammen von Regierungen und Hilfsorganisationen sind Mutter-Kind-Programme, in denen den Frauen gezeigt wird, wie sie eine ausgeglichene Ernährung zubereiten können. Wenn die Regierungen aber den Akzent auf Grossprojekte statt auf eine Förderung der Subsistenzwirtschaft setzen, und die Preise für die Grundnahrungsmittel so hoch sind, dass die Bevölkerung in den Städten sie nicht kaufen kann, sind zwar solche Gesundheitsprogramme gut gemeint, aber unrealistisch. Es kommt hinzu, dass die Regierungen ihr Staatsbudget infolge der Verschuldung immer zuerst im Bereich der Sozialausgaben kürzen und der Zugang zur Gesundheitsversorgung und zur Bildung für die Bevölkerung und besonders für die Frauen immer schwieriger wird.

Das Thema "Frauen und Entwicklung» kann nicht losgelöst behandelt werden und darf sich nicht auf die Förderung von Frauenprojekten oder Frauenkomponenten in integrierten Entwicklungsprojekten beschränken, sondern muss in einem globalen nationalen und internationalen Rahmen angegangen werden. Seit den achtziger Jahren wird denn auch der Begriff «Integration der Frauen in die Entwicklung" stark kritisiert. Am NGO-Forum in Nairobi, das anlässlich der UN-Weltfrauenkonferenz stattfand, haben Dritt-Welt-Frauenorganisationen wie DAWN, Philipina, AAWORD, ZARD, WIN (2) den Einbezug von Frauen in Entscheidungsprozesse auf allen Ebenen und die Definition und Durchführung von neuen Entwicklungsstrategien gefordert, welche die Demokratisierung der Regierungen und eine gerechtere Weltwirtschaftsordnung zum Ziel haben. Ohne Erfüllung dieser Bedingung ist ein emanzipatorischer Prozess der Frauen ausgeschlossen. In einem Manifest haben die am Forum anwesenden afrikanischen Frauen alternative Entwicklungsstrategien für die Entwicklung in Afrika aufgestellt (3).

Mir scheint bei der Auseinandersetzung über eine frauengerechtere Entwicklung in Afrika wichtig, die Frauenproblematik im Zusammenhang mit der nationalen Entwicklung der afrikanischen Staaten anzusehen. Afrikanische Autorinnen, wie z.B. Achola Pala (4) weisen darauf hin, dass der Entwicklungsprozess seit der Kolonialzeit nicht nur auf die Frauen negative Auswirkungen hatte, sondern auch für die Männer nicht positiv war. Denkt man an die Situation der Subsistenzbauern oder an die vielen Arbeitslosen in Städten wie Dakar, so leuchtet dieser Einwand ein. Natürlich steht auch fest, dass es gerade in den «ärmsten» Bevölkerungsschichten den Frauen noch eine Stufe schlechter geht. Anzustreben ist ein Emanzipationsprozess der ganzen Gesellschaft, in dem Frauen und 
Männer eine komplementäre und gleichberechtigte Rolle spielen. Dies bedingt ein Umdenken bei den Männern, weil ohne wirkliche Veränderung von soziokulturellen Strukturen und den gegebenen Macht- und Besitzverhältnissen, welche die Frauen unterdrücken, keine gerechtere Gesellschaftsform erreicht werden kann.

Wie lassen sich Theorie und Praxis miteinander verbinden? Was für eine Rolle spielen Entwicklungsprojekteprogramme im Emanzipationsprozess der Sahelländer und hier spezifisch im Befreiungsprozess der Frauen? Welches ist die Rolle kirchlicher Hilfswerke, welche ihren Auftrag im Christentum und im Evangelium begründen und als "Anwalt der Armen" ungehindert von eigenen politischen und wirtschaftlichen Interessen klar für Frieden, Gerechtigkeit und Befreiung von Abhängigkeit und diskriminierenden Verhältnissen eintreten?

Die Beziehung des HEKS zu einem grossen Teil seiner Sahelpartner geht auf die Dürrekatastrophe von 1973/74 zurück. Als Antwort auf den Appell für internationale Hilfe haben die Hilfswerke der ökumenischen Familie, zu denen auch das HEKS gehört, über den Ökumenischen Rat der Kirchen eine umfassende Nothilfeaktion durchgeführt. Aus dem Nothilfeprogramm entwickelte sich ein Solidaritätsprogramm zwischen den ökumenischen Hilfswerken, den afrikanischen Kirchen und den Basisgruppen und lokalen Hilfsorganisationen in den acht Sahelländern. Unterstützt werden ganz konkrete Initiativen zur Förderung der Nahrungsmittelproduktion im Bereich der Landwirtschaft, der Viehzucht und der Fischerei, Wiederaufforstungsprogramme und Massnahmen zum Schutz der Böden, Verbesserung der Wasserversorgung, sowie Aufbau von Basisgesundheitsdiensten. Als wichtiger Schwerpunkt zur Stärkung der zahlreichen dörflichen Basisgruppen und Selbsthilfegruppen werden vor allem Ausbildungsprogramme gefördert: Ausbildung von dörflichen Berater/Innen, Austauschprogramme zwischen Basisgruppen, welche in einem ähnlichen ökologischen Kontext leben, Workshops über technische sowie bewusstseinsbildende Themen. Neben dem ökumenischen Solidaritätsprogramm unterstützt das HEKS auch andere Partnerorganisationen bilateral: lokale NGO's, kirchliche Partnerorganisationen und Basisinitiativen. Gemeinsames Ziel all dieser Entwicklungsprogramme und Gemeinschaftsinitiativen ist eine ganzheitliche Verbesserung der Lebensverhältnisse der Bevölkerung ausgehend vom Vertrauen auf die eigenen Kräfte und dem Gebrauch der eigenen Ressourcen. Im Gegensatz zu einer wachstumsorientierten Entwicklung geht es hier um die Stärkung der Subsistenzwirtschaft: die Förderung einer integrierten und umweltschonenden Landwirtschaft, die Konzentration auf die Binnenmärkte und auf die traditionellen Nachbarschaftsbeziehungen.

Die Wichtigkeit des Einbezugs der Frauen in die Planung und Durchführung von Entwicklungsprojektenprogrammen und der Integration der Frauen in die Entscheidungsmechanismen wird seit den achtziger Jahren zunehmend von allen Partnerorganisationen erkannt. In fast allen Entwicklungsprojekten gibt es ein Frauenprogramm, das den speziellen Bedürfnissen der Frauen Rechnung trägt. Innerhalb des ökumenischen Sahelprogramms wurden in den letzten zehn 
Jahren zahlreiche Frauengruppen und Frauenaktivitäten unterstützt. 1986 wurde ein Seminar über die Möglichkeiten und Grenzen der Frauen im Entwicklungsprozess der Sahelländer organisiert, wo die Frauen - zwar noch zaghaft und in einer allgemeinen Form - ihre Vorstellungen von frauenfördernden Massnahmen in der Entwicklungszusammenarbeit darstellten. Der Bewusstseinsgrad in bezug auf das Thema "Frauen und Entwicklung" und die praktische Ausrichtung sind im einzelnen Fall jeweils anders, je nach Region und Konstellation des Programms. Sozio-kulturelle und religiöse Faktoren, sowie die staatliche Regierungspolitik spielen dabei eine bedeutende Rolle.

\section{Frauen in konkreten Entwicklungsprojekten}

Anhand von zwei Beispielen soll aufgezeigt werden, wie "Frauen und Entwicklung" in der Praxis gelebt und verwirklicht wird.

Das erste Beispiel, welches für meine Projektarbeit repräsentativen Charakter hat, wegen seiner wenig idealen Bedingungen, wie Bürgerkrieg, Dürrekatastrophen, Heuschrecken, Überschwemmungen etc., stammt aus dem Tschad. In diesem Land verfolgt die Regierung in Sachen Frauenförderung noch keine gezielte Politik.

\section{Das «Centre Horticole et d'Animation Rurale, Bougoumène» (CHARB)/Tschad:}

Das landwirtschaftliche Beratungszentrum in Bougoumène (5) (CHARB) entwickelte sich aus einer lokalen Initiative der "Eglise de Dieu» heraus, die darin bestand, 1517jährige Schulabgänger der Primarschule Bougoumène und Umgebung im Gemüse- und Getreideanbau auszubilden. In der Zeitspanne von 1976-80 wurden fünfundvierzig junge Leute ausgebildet. Als sich die Lage im Tschad durch anhaltende Dürreperioden und den Bürgerkrieg zusehends verschlechterte, weitete die Landwirtschaftsschule ihren Arbeitsbereich stark aus. Die Landwirtschaftsschule sollte nicht nur einer kleinen Gruppe von Privilegierten die Möglichkeit einer Ausbildung bieten, sondern grundsätzlich allen Bauern und Bäuerinnen der Region offenstehen. Im Vordergrund stand fortan die Ausbildung und Beratung von Dorfgruppen. In enger Zusammenarbeit mit früheren Schülern, die nach Abschluss der Ausbildung in drei Dörfern Gemüsekulturen anlegten, wurden weitere Bewässerungsprojekte geschaffen. Es entstanden neue Aktionsbereiche wie Kollektivfelder, Getreidespeicher, Fischerei- und Kleinprojekte, Dorfapotheken. Heute umfasst das CHARB ein weites Gebiet längs des Chari- und Logone-Flusses und berät an die achtzig Basisgruppen. Die Arbeit im CHARB leisten der verantwortliche Leiter des Beratungszentrums, sieben Animatorinnen und ein Animator.

Neben den Gemüsekulturen bilden die Frauenaktivitäten den Schwerpunkt in der Arbeit des landwirtschaftlichen Beratungszentrums. 1984/85, als die Lage der Bevölkerung und besonders der Frauen und Kinder äusserst dramatisch war, hat das CHARB den Sektor der "animation féminine" wesentlich verstärkt. Zu dieser Zeit bestand die Arbeit der Animatorinnen vor allem in der Nothilfe durch die Durch- 
führung von Ernährungsprogrammen für Mütter und Kinder: Verteilung von Lebensmitteln, Vorführung von "bouillies enrichies" für Kleinkinder, erste Hilfe und Beratung der Frauen in Gesundheitspflege und Hygiene. Mit der Stabilisierung der Situation verlegte sich die Priorität frauenspezifischer Aktivitäten auf andere Bereiche wie Kleinhandel, Viehzucht, Getreidemühlen, Kollektivfelder, Gemüsegärten und Nähateliers.

Aufgrund einer Evaluation der Frauenaktivitäten des CHARB 1986, welche im Rahmen einer Studie über Dorfinitiativen durchgeführt wurde (6), der Jahresberichte 1986 und 1987, sowie meines kurzen Feldbesuchs im Februar 1987 lässt sich folgendes Bild von den Frauenaktivitäten des CHARB zeichnen:

Für die Frauen im Gebiet von Bougoumène ist es neu, sich in Gruppen zu organisieren. Die Gruppen sind noch nicht sehr gut strukturiert, und der Selbständigkeitsgrad der Frauen innerhalb der Gruppe ist in den Dörfern noch gering. Während die Animatorinnen vor allem in den Bereichen der Gesundheit (Dorfapotheken, Kinderpflege, Ernährungslehre, Hygiene) und der Nähateliers mit den Frauen arbeiten, also für die "typischen" Frauenaktivitäten im Sozialwesen verantwortlich sind, werden die Projekte, die den landwirtschaftlichen Sektor betreffen, wie Gemüsegärten, Getreidespeicher, Viehzucht und Mühlen, vom Leiter des CHARB und einem Animator begleitet. Je nach Art der Aktivitäten sind die Gruppen geschlechtsspezifisch zusammengesetzt. Gemüsekulturen und Getreidespeicher werden von Männern und Frauen betrieben, wobei der Anteil der Männer höher ist. Die Aufzucht von Schafen und Ziegen obliegt den Frauen und jene der Rinder den Männern. Die Installation von Mühlen wird als arbeitserleichternde Massnahme für Frauen betrachtet. Die Zielgruppen dieser Projekte sind folglich Frauengruppen. Im Verwaltungskomitee sind Frauen und Männer vertreten; die Hauptfunktionen wie Verwaltung, Buchhaltung, Rentabilitätsrechnung werden aber von Männern ausgeführt. Die Frauen sind aus den Verantwortungs- und Entscheidungsprozessen ausgeschlossen.

Es gibt aber Aktivitäten, wo sich die Frauen ganz selbständig zusammengeschlossen und organisiert haben und diese Aktivitäten autonom durchführen. Dies sind die Bestellung von Kollektivfeldern und der Kleinhandel.

Einen ganz besonderen Platz in den Frauenaktivitäten nehmen die Nähateliers ein. In einer Umfrage im Rahmen der Evaluation haben fünfzehn von zwanzig Frauengruppen die Nähateliers auf den ersten Rang ihrer Prioritätenliste gesetzt. Auch von den Animatorinnen werden den Nähateliers Priorität eingeräumt. Frauen und junge Mädchen nähen und sticken für den Eigengebrauch und für den Verkauf im Dorf oder in der Stadt. Der Erlös aus dem Verkauf kommt in eine Gemeinschaftskasse. Dieses Geld dient zur Anschaffung von neuem Material oder von Medikamenten, Schulmaterial für die Kinder und Gebrauchsartikeln.

Zusammenfassend kann gesagt werden, dass die Frauenaktivitäten im CHARB keine echte Veränderung der Machtverhältnisse und der Rollenverteilungen bringen, sondern eher einen integrativen Prozess auf der Basis von traditionellen Macht- und Rollenzuteilungen darstellen. Die Frauen sind in den Entscheidungsmechanismen sowohl auf der Projektebene als auf der Ebene des Zentrums und der Trägerorganisation nicht oder nur formell vertreten.

- Aktivitäten wie “Nähen und Sticken» sind ökonomisch nicht interessant, weil schlecht bezahlt, und zementieren das traditionelle Rollenbild Frau/Mann. 
- Die Aktivitäten innerhalb des Gesundheitsprogramms wie Dorfapotheken, Ernährungslehre und Hygiene bringen nicht eine wesentliche Verbesserung im Gesundheitswesen, weil sie nicht in ein nationales, globales Programm integriert sind.

- Im landwirtschaftlichen Bereich können Verbesserungen der Lebenssituation der Frauen festgestellt werden. Der Anbau von Gemüse und die Möglichkeit, einen Kredit für den Kauf von ein paar Ziegen und Schafen aufzunehmen, ermöglichen eine bessere Ernährung der Bevölkerung. Dies hat eine direkte Auswirkung auf deren Gesundheit.

- Mühlen erleichtern den Frauen die Arbeit, vorausgesetzt, dass genügend Hirse zum Mahlen vorhanden ist und die Frauen den Preis fürs Mahlen bezahlen können, was in der Region von Bougoumène nicht überall der Fall ist.

Was aber am meisten auffällt, ist das Gefälle zwischen dem CHARB und den Basisgruppen in allen Aktionsbereichen. Die Animationsmethode der Beratungsgruppe bewirkt keine partizipative und selbstbestimmende Haltung der Frauen und Männer in der Durchführung der Projekte. Sowohl in den frauenspezifischen Aktivitäten als in den anderen Projekten besteht die Beratung in der Demonstration von Wissen. Eine wirkliche Animation im Sinne einer Stimulierung und Anleitung der Gruppen, ihre Situation zu analysieren und entsprechende Lösungen zu finden und für ihre Aktivitäten selber die Verantwortung zu übernehmen, fehlt noch weitgehend.

$\mathrm{Zu}$ den Frauenaktivitäten muss noch hinzugefügt werden, dass die Sensibilisierungsarbeit mit den Frauen vor allem am Anfang sehr schwierig war, weil die Frauen in dieser Region verschiedenen Ethnien angehören und in einem ganz unterschiedlichen sozialen, kulturellen und religiösen Umfeld leben. Über die Nähateliers wurde die Arbeit mit den Frauen erst möglich. Auch scheinen die Frauen sehr gerne zu nähen. Sie fehlen fast nie in den wöchentlichen Versammlungen. Diese Versammlungen bilden für die Frauen einen Freiraum, wo sie sich treffen können. Sie können eine gute Basis bilden, auf der weitere Massnahmen aufgebaut werden.

Unterstützen kann meiner Ansicht nach den emanzipatorischen Prozess der Frauen eine gute nichtformale Ausbildung, z.B. bewusstseinsbildende Seminare und SüdSüd-Austausch mit anderen Frauengruppen. Ein Schritt in diese Richtung sind auch Autoevaluationen, die zugleich Ausbildungsfunktion haben für die Basisgruppen und für das beratende Personal des CHARB. In diesem Sinne wurden die Bewässerungsprojekte und die Getreidebanken mit der Unterstützung von INADES-FORMATION evaluiert. Wichtig ist eine kontinuierliche Ausbildung und Unterstützung.

Das zweite Beispiel kommt aus Burkina Faso. Im Gegensatz zum Tschad und anderen Sahelländern hat die Regierung in Burkina Faso eine klare frauenfördernde Politik definiert. Eines der Hauptziele des Aktionsprogramms des «Front Populaire» ist die Unterstützung der Frauen in ihrem Emanzipationsprozess. Die Frauen sollen längerfristig in die Entscheidungsstrukturen der Regierung auf allen Ebenen einbezogen werden. In sein Fünfjahresprogramm 1986-1990 hat der «Front Populaire» ein Aktionsprogramm für die Frauen integriert.

Zur Förderung von einkommensschaffenden Projekten für die Frauen wurden verschiedene konkrete Massnahmen durchgeführt. Unter dem Motto «faso dan fani" und "consommer burkinabé" lancierte die Regierung eine Initiative, 
um die nationale Produktion in Landwirtschaft und Handwerk und die Vermarktung der Produkte zu fördern. Der Import von Gemüse, Früchten und Luxusgütern aus dem Ausland wurde verboten und ein Dekret erlassen, nach dem alle Staatsangestellten Kleider aus handgewebten Stoffen tragen müssen, die von den Frauen Burkinas produziert werden.

Die «Regierung Sankara» (7) hat ohne Zweifel einen Befreiungsprozess für die Frauen in Gang gesetzt, der auch weiterhin stattfindet, obwohl die staatlichen Frauenorganisationen im Moment in einer Krise sind und sich überlegen, wie sie sich neu definieren und strukturieren wollen. Die neue Regierung ist im Zeichen der «rectification» für Initiativen wie «faso dan fani» nicht mehr sehr offen, und der Schwerpunkt des Aktionsprogramms liegt nicht mehr auf der Mobilisierung und Unterstützung einer Volksbewegung.

\section{Das Entwicklungsprogramm der FEME (Fédération des Eglises et Missions Evangéliques)}

Die FEME umfasst die sieben nationalen evangelischen Kirchen und Missionen Burkina Fasos. 1972 hat der evangelische Kirchenbund ein Projektbüro eingerichtet, um an der sozialen, wirtschaftlichen und kulturellen Entwicklung des Landes teilzuhaben. In ihrer Arbeit orientiert sich die FEME an den Grundbedürfnissen der Bevölkerung; ihre Hauptunterstützung gilt der ländlichen Bevölkerung. In den ersten zehn Jahren lag der Schwerpunkt vor allem auf der Nothilfe. Seither entwickelte sich das Aktionsprogramm immer mehr in Richtung eines integrierten Entwicklungsprogramms mit dem Ziel einer globalen Verbesserung der Lebensbedingungen der Bevölkerung in den Bereichen Landwirtschaft (Ackerbau, Getreidebanken, Mühlen, Wiederaufforstung, Viehzucht), Hydraulik (Rückstaubecken, Brunnen), Gesundheit und Erziehung (Schulen, Ausbildung von Handwerkern/Innen). Durch eine breit abgestützte Struktur ist es der FEME möglich, wirklich an der Basis zu arbeiten und eine selbstbestimmte und partizipative Entwicklung der Basisgruppen zu fördern. In den Dörfern arbeiten 53 Koordinatoren und 54 Koordinatorinnen mit den Bauernund Frauengruppen. Das operationelle Team, in das auch ein technischer Dienst integriert ist, ist für die Ausführung, Vorabklärung und Realisierung der Projekte verantwortlich. Das Entwicklungsprogramm der FEME erstreckt sich über das ganze Land und ist im Fünfjahresplan der Regierung integriert.

\section{Frauenabteilung}

Dem Projektbüro gehört eine Frauenabteilung an. Eine Evaluation der Aktivitäten der FEME nach zehnjährigem Bestehen hatte ergeben, dass die bestimmende Rolle der Frauen im Entwicklungsprozess der ländlichen Bevölkerung nicht wahrgenommen worden war. Bis anhin hatten die Frauen nur am Rande von der Unterstützung der FEME profitiert und wurden weder in die Planung und Durchführung von Projekten miteinbezogen, noch wurden die sozio-kulturellen und wirtschaftlichen Auswirkungen von Projekten auf die Frauen untersucht. Die Schaffung einer Frauenabteilung, welche auf die spezifischen Bedürfnisse der Frauen ausgerichtet ist und die Entwicklung der Aktivitäten des Projektbüros auch aus der Perspektive der Frau be- 
leuchtet, war dringend nötig. In einer Zeitspanne von zwei Jahren wurde die Frauenabteilung aufgebaut.

Vom Inhalt her unterscheiden sich die frauenfördernden Projekte der FEME nicht wesentlich von denjenigen anderer privater Organisationen. Es sind einerseits einkommensschaffende Projekte, wie z.B. Kollektivfelder, Kleintierzucht, Getreidebanken, Gartenbau, Herstellung von Seifen und Karité-Butter, Mehl, Kuchen für den Kleinhandel, Weben, Färben, Herstellung von Körben, Töpfen. Andererseits sind es soziale Projekte, die der Arbeitserleichterung der Frauen oder einer sozialen Verbesserung der Dorfstruktur dienen, wie z.B. Mühlen, Hirse- und Getreideschälmaschinen, Trinkwasserversorgung, Gesundheitszentren, Kindergärten. Was jedoch auffällt, ist das Engagement der Mitarbeiter des Projektbüros wie auch der Kordinatoren/Innen im Feld, welche sich mit Herz und Seele für die Entwicklung ihres Landes einsetzen. Die Koordinatorinnen der Frauenabteilung gehen immer von den Bedürfnissen und der Situation der Frauen aus. Die Initiative muss von den betroffenen Frauen selber kommen. Die Koordinatorinnen helfen den Frauen, ihre Situation besser zu verstehen und gemeinsam Verbesserungen in ihrem Arbeits- und Lebensbereich anzustreben. Sehr viel Wert wird auf die Aus- und Weiterbildung der Koordinatorinnen und Verantwortlichen der Frauengruppen gelegt. So werden jedes Jahr verschiedene Seminare über Animationstechniken und spezifische Fachbereiche organisiert. Im vergangenen Jahr fanden mehrere Seminare zum Thema "einkommensschaffende Projekte" statt. Konkret ging es um die Webeateliers und den Kleinhandel. Im ganzen haben 524 Frauen an diesem Weiterbildungsseminaren teilgenommen. Solche Seminare helfen den Frauen, sich besser zu organisieren, ihre Tätigkeit technisch besser zu meistern und die Marktmechanismen zu durchschauen. Längerfristiges Ziel der FEME ist eine grössere Autonomie der Frauengruppen und die Bildung von Kooperativen.

Der Grad der Autonomie und Partizipation der Frauengruppen, die von der FEME in 54 Distrikten begleitet werden, ist sehr unterschiedlich. Es gibt Frauen, die erst anfangen, sich zu organisieren, und Frauengruppen, die ganz selbständig funktionieren.

\section{Süd-Süd-Austausch}

Eine andere Art von Ausbildung, welche die FEME stark fördert, ist der Süd-SüdAustausch. Frauengruppen aus verschiedenen Regionen Burkina Fasos oder aus anderen Sahelländern, welche in einem bestimmten Bereich, wie z.B. in der Kommerzialisierung und Konservierung von landwirtschaftlichen Produkten, unterschiedliche Erfahrungen gemacht haben oder eine Tätigkeit mehr oder weniger gut beherrschen, besuchen einander gegenseitig und übermitteln Wissen und Erfahrungen.

Es bleibt auch innerhalb der FEME in Sachen Frauenförderung noch viel zu tun. Die Frauen sind zwar auf der Projektebene in die Entscheidungsstrukturen einbezogen. Die Frauengruppen verwalten ihre Aktivitäten selber. In gemischten Basisgruppen sind auch Frauen im Verwaltungskomitee vertreten. Auf der Ebene des Projektbüros ist die Frauenabteilung zu einem grossen Teil autonom und wird auch bei der Planung von anderen Projekten miteinbezogen, so z.B. beim Bau eines Rückstaubeckens in die Phase der sozio-kulturellen und ökonomischen Vorstudie, sowie in die Planung der Nutzung der Grundstücke, welche durch das Rückstaubecken bewässert werden. Auf höherer Ebene sind die Frauen jedoch nicht mehr vertreten. 
Der Verwaltungsrat des Projektbüros, welcher die Entscheidungen über die Politik sowie die Durchführung von Projekten trifft, setzt sich ausschliesslich aus Männern zusammen. Der Verantwortliche des Projektbüros ist ebenfalls ein Mann. Er hat die Entwicklungsarbeit der FEME aufgebaut, und auch heute noch laufen alle Fäden bei ihm zusammen. Doch hat die Frauenabteilung eine eigene Dynamik und Autonomie entwickelt und könnte heute nicht mehr weggedacht werden.

Zur Illustration von Frauenförderung in der konkreten Projektarbeit wurden zwei sehr unterschiedliche Beispiele gewählt: Einerseits das CHARB, eine kleine lokale Organisation im Tschad, welche neben wiederholten Dürre- und Hungerperioden einem grausamen zehnjährigen Bürgerkrieg ausgesetzt war, andererseits das Enwicklungsprogramm der FEME, ein sehr grosses Projekt, das jährlich Millionenbeträge verwaltet und eine globale und integrierte Entwicklung in allen Provinzen von Burkina Faso verfolgt. Die Projekte sind von ihrer Struktur, ihren Kapazitäten und ihrem Potential, wie auch vom politischen und sozio-kulturellen Umfeld her unterschiedlich. Die Beispiele wurden bewusst so gewählt, um das Thema, das zu einer Art Modethema geworden ist, nicht zu verschönern, oder es bei generellen Aussagen bewenden zu lassen.

Spätestens seit dem Abschluss der UN-Frauendekade in Nairobi ist das Bewusstsein für eine frauengerechtere Entwicklung überall erwacht. Der Weg zu einer gerechteren Gesellschaft, wie sie von den Dritt-Welt-Frauen am NGOForum in Nairobi definiert wurde, durchläuft jedoch unterschiedliche Stadien der Konkretisierung.

\section{«Frauen und Entwicklung» in der Projekt-Sachbearbeitung}

Auch nach dem Abschluss des Projektes «Frauen und Entwicklung", welches von verschiedenen privaten Schweizer Hilfswerken - darunter auch das HEKS - finanziert und mitgetragen wurde, besteht weiterhin eine Arbeitsgruppe, die sich speziell mit dem Bereich "einkommensschaffende Massnahmen» auseinandersetzt. Innerhalb dieser Gruppe haben sich Untergruppen nach Kontinenten gebildet. In der Afrika-Gruppe beispielsweise treffen sich in regelmässigen Abständen zwei Frauen (die Autorin als Vertreterin des HEKS und eine freischaffende Frau) sowie ein Vertreter des SAH, um die von innen bearbeiteten Projekte näher auf den Aspekt «Frauen und Entwicklung» zu analysieren und mit den Frauen in den Projekten einen partnerschaftlichen Dialog aufzubauen, in den auch die Situation der Sachbearbeiterin hier einbezogen wird. In Ländern, in denen sowohl das SAH als auch das HEKS arbeiten, versucht die Gruppe, einzelne Fraueninitiativen miteinander zu vernetzen. Ein Anliegen ist auch, einzelne konkrete Massnahmen, wie z. B. ein Mühleprojekt, Gemüsegärten, Kollektivfelder, sozusagen als Fallbeispiele eingehender zu verfolgen, weil oft genaue Informationen über die einzelnen Projekte fehlen und die Leute in der Zentrale nicht wissen, inwieweit die Projekte tatsächlich einkom- 
mensschaffend sind und die Autonomie der Frauen fördern. Die Mitglieder der Arbeitsgruppe versuchen weiter, durch die Lektüre von Monographien und sozio-ökonomischen Studien über die Situation der Frauen den Hintergrund der Projekte kennenzulernen. Im Bewusstsein, nur sehr beschränkt am Thema «Frauen und Entwicklung» arbeiten zu können, versuchen sie beharrlich, neben der Überlastung und anderen Sachzwängen in der Arbeit, diesen Freiraum auch in Zukunft zu erhalten.

Innerhalb des Entwicklungsdienstes des HEKS ist "Frauen und Entwicklung" ein Themenbereich neben anderen inhaltichen Schwerpunkten wie z.B. "Verschuldung", "Ökologie", "Verletzung von Menschenrechten». Von fünf Sachbearbeitern setzen sich zwei im Rahmen der Arbeitsgruppe besonders mit dem Thema "Frauen und Entwicklung" auseinander. Eine Mitarbeiterin des Sekretariats ist für die Koordination und die Dokumentation verantwortlich, kann aber diese Aufgabe infolge Arbeitsüberlastung nur sehr beschränkt wahrnehmen.

Das Bewusstsein ist sicher auf allen Ebenen da, und jede/r Mitarbeiter/in hat das Thema auf eigene Weise verinnerlicht; doch befindet sich auch das HEKS in einem Prozess der Weiterentwicklung. Das Thema muss immer wieder und überall eingebracht werden, denn "Frauen und Entwicklung» betrifft alle Lebens- und Arbeitsbereiche. Die Frage der Frauenförderung darf sich nicht auf die Projektarbeit beschränken, sie muss in unsere Denkmodelle, in unsere Handlungen und Strukturen einfliessen.

\section{Anmerkungen und Quellen}

1) Mauretanien, Senegal, Gambia, die Kapverdischen Inseln, Mali, Burkina Faso, Niger, Guinea Bissau und Tschad

2) Development Alternatives with Women for a New Era, Philipina, eine nationale philippinische Frauenorganisation, Association of African Women for Research and Development, Zambia Association for Research and Development, Women in Nigeria

3) vgl. AAWORD: “AAWORD in Nairobi, 85", Occasional Paper Series No 3

4) Achola Pala: «Definitions of Women and Development: An African Perspective. In : Women and National Development: The Complexities of Change»

5) Bougoumène liegt etwa $80 \mathrm{~km}$ südlich von N'Djaména am Chari-Fluss

6) Geneviève Pillet, INADES-FORMATION, Tchad, Evaluation-auto-évaluation des activités du Centre horticole et d'animation rurale de Bougoumène. Activités féminines, 1986

7) Thomas Sankara war von 1983-1987 Präsident von Burkina Faso. Er kam am 15. Oktober 1987 bei einem Militärputsch ums Leben. 